\title{
Cultural Diversity Broadens Social Networks
}

\author{
Adrienne Wood $^{1 *}$, Adam M. Kleinbaum ${ }^{2}, \&$ Thalia Wheatley ${ }^{3}$
}

Full citation: Wood, A., Kleinbaum, A. M., \& Wheatley, T. (2022). Cultural diversity broadens social networks. Journal of Personality and Social Psychology.

https://doi.org/10.1037/pspi0000395

\section{Author Note}

*Corresponding author: Adrienne Wood, 1-913-907-9370, adrienne.wood@virginia.edu.

1. Department of Psychology, University of Virginia, 485 McCormick Rd, Charlottesville, VA, 22904.

2. Tuck School of Business, Dartmouth College, 100 Tuck Hall, Hanover, NH, 03755.

3. Department of Psychological and Brain Sciences, Dartmouth College, 6207, Hanover, NH, 03755 .

The population-level variables and code for data processing and analysis are available on OSF (https://osf.io/ycpwt). The analysis output is viewable on RPubs (https://rpubs.com/adriennewood/cultural-diversity). We do not have permission to share participant-level data publicly. 


\begin{abstract}
Migration and mobility increase the cultural diversity of a society. Does this diversity have consequences for how people interact and form social ties, even when they join a new community? We hypothesized that people from regions with greater cultural diversity would forge more diversified social ties in a newly formed community, connecting otherwise unconnected groups. In other words, that they would become social brokers. We tested this prediction by characterizing the social networks of eight Master of Business Administration cohorts $(\mathrm{N}=2,257)$ at a business school in the U.S. International students $(\mathrm{N}=773)$ from populations with both greater present day ethnic diversity and a history of extensive cultural intermingling were more likely to become social brokers than international students from less diverse nations. Domestic students' $(\mathrm{N}=1,461)$ brokerage scores were also positively related to the ancestral diversity of the U.S. county they identified as "home." The results of this study suggest that more culturally diverse social environments - defined here at multiple geographic and temporal scales — endow people with socially adaptable behaviors that help them connect broadly within new, heterogeneous communities.
\end{abstract}

Keywords: social networks, diversity, culture, social brokerage, socio-ecology 


\section{Cultural Diversity Broadens Social Networks}

People tend to befriend similar others (McPherson et al., 2006). This rule of homophily in networks promotes the development of tight social clusters wherein people are insulated from differences - for example, a clique of high school students who only socialize within their group or members of a work team who rely exclusively on each other for social support. Others build social networks that are less constrained, becoming "social brokers" (Burt, 1992) in a network by connecting with numerous people from different groups. This diverse connectivity creates bridges between subcommunities, giving social brokers greater access to a broader range of the network's resources (Erickson, 2003), enabling the exchange of ideas and resources that benefit the whole community (Carnovale \& Yeniyurt, 2015; Chua et al., 2015; Derex \& Boyd, 2016) and facilitating innovation and creativity (Burt, 2004; Muthukrishna et al., 2014; Muthukrishna \& Henrich, 2016; Perry-Smith \& Shalley, 2003). Brokers can also flexibly recruit different people to provide information and social support, depending on their needs. Much like diversifying a stock portfolio to protect against financial risk, these broad connections hedge against future social isolation, should a tie to a particular person or subgroup become severed (Cattell, 2001; Karsai et al., 2014). Although any form of social connection may be beneficial (Yang et al., 2016), brokerage is associated with better mental health (Erickson, 2003; Mitchell \& LaGory, 2002), physical health (Putnam, 2000), and career outcomes (Burt 1992; Soda et al 2004).

By definition, one who becomes a social broker must have the motivation and capacity to interact with many different types of people. Brokers thus are remarkably adaptable, whether due to having the social bandwidth for connecting with people who are measurably different from them or the ability to discover what they do have in common with new social partners (Hong et 
al., 2000). Some communities may demand more social adaptability than others-for instance, culturally diverse communities, in which interacting with many types of people is a fact of life. The present work asks whether experience in a culturally diverse social environment prepares people to play the role of social broker in future social networks.

\section{Diverse socio-ecologies encourage social adaptability}

Recent work suggests that cultural diversity - the amount of historical and present-day migration, population flux, and cultural exchange with other populations (Niedenthal et al., 2017) — may compel norms related to social adaptability. These norms include greater flexibility to make and break social ties (Thomson et al., 2018), greater trust in strangers, openness (Shrira et al., 2018), and nonverbal expressivity (Niedenthal et al., 2019). The resultant social adaptability may afford individuals greater access to resources in a heterogenous social environment (Eagle et al., 2010; Morrison, 2002; Putterman \& Weil, 2010). Consistent with cultural diversity promoting brokerage, Burt (2019) argues that access and exposure to diversity equips social brokers to be successful entrepreneurs because they can more flexibly adapt to the demands of a new situation.

One way to describe differences in cultural diversity across populations is on a longhistory timescale. As an example, Argentina's ancestrally-diverse population resulted from significant waves of migration from 37 different nations since the year 1500 (estimated by Putterman \& Weil, 2010 using archival and genetic records). The history of an ancestrally diverse population like Argentina involves repeated co-mingling of cultures. Between-group cooperation in such an environment likely required social adaptability to overcome the barriers of cultural and linguistic differences. In contrast, ancestrally homogeneous places like Ethiopia have, over the past few centuries, experienced low levels of immigration or cultural mixing, 
giving social norms and structures time to stabilize. In such environments, we speculate that excessive social adaptability is less useful (Geeraert et al., 2019), and may even be maladaptive by increasing the likelihood of social norm violations. Indeed, ancestral diversity is associated with looser social norms (Gelfand et al., 2017; Niedenthal et al., 2019) as well as higher relational mobility — in such societies, social ties are more transient and relationships can be formed and ended more easily (Thomson et al., 2018). Thus, even though the populations of ancestrally diverse societies may have since stabilized to resemble a more homogeneous population, they appear to retain traces of their historical diversity in their norms and social structures.

Recent studies suggest that populations with a long history of immigrant commingling display traits that enable flexible social connection (Niedenthal et al., 2019). People in ancestrally diverse societies tend to be higher on openness to new experiences (Shrira et al., 2018), a personality trait that, in turn, is correlated with having diverse connections in a social network (Fang et al., 2015). Moreover, people living in ethnically diverse subcommunities report greater life satisfaction if they are higher in openness to new experiences (Jokela et al., 2015). The correlation of ancestral diversity with relational mobility and normative looseness leads to, respectively, greater self-expression (Thomson et al., 2018) and self-monitoring (Gelfand et al., 2011), respectively. In other words, people from ancestrally diverse populations do more work, on average, to advertise their social value and adjust to the demands of new interaction partners. People from ancestrally culturally diverse populations are also more nonverbally expressive, especially for positive affective displays like smiles (Girard \& McDuff, 2017; Niedenthal et al., 2018, 2019). Their nonverbal displays are more cross-culturally recognizable than those of homogeneous populations (Wood et al., 2016), presumably a vestige of having to facilitate 
communication in the absence of a shared language and background. Thus people from historically diverse populations_-populations that have acted as group-level cultural "brokers" over centuries - display traits like expressiveness and openness that are associated with social adaptability (Niedenthal et al., 2019).

Finally, indirect self-report evidence suggests exposure to mobile and diverse socioecologies prepares people to form social ties in new environments. Student migrants from more relationally mobile nations report lower levels of loneliness than do migrants from nations with lower average relational mobility scores (Heu et al., 2020). This finding suggests prior socioecologies might shape people's social network positions in new communities.

\section{Does exposure to diverse socio-ecologies predict whether people become social brokers?}

In the present work, we asked whether growing up in a culturally diverse community enables people to become social brokers in a new and diverse social network. To answer this question, we characterized the social networks of first-year Master of Business Administration (MBA) students across eight cohorts and predicted their social brokerage scores from estimates of their home community's cultural diversity (Figure 1). Note that cultural diversity, and diversity more generally, can be quantified in many ways, so the following variables do not capture the full scope of human diversity.

For international students, our two measures of cultural diversity at the nation level capture longterm historical migratory trends and more recent demographic changes. For the former, we included ancestral diversity, or the diversity of the source nations that contributed significantly to the nation's population through (forced or voluntary) immigration over the last 500 years. Measures of ancestral diversity computed from the World Migration Index (Putterman \& Weil, 2010) predict a number of present-day behavioral and trait measures in prior work, as discussed 
earlier (Niedenthal et al., 2019). To quantify the present-day cultural diversity of international students' home nations, we used a widely-researched nation-level index of ethnic fractionalization (Alesina et al., 2003).

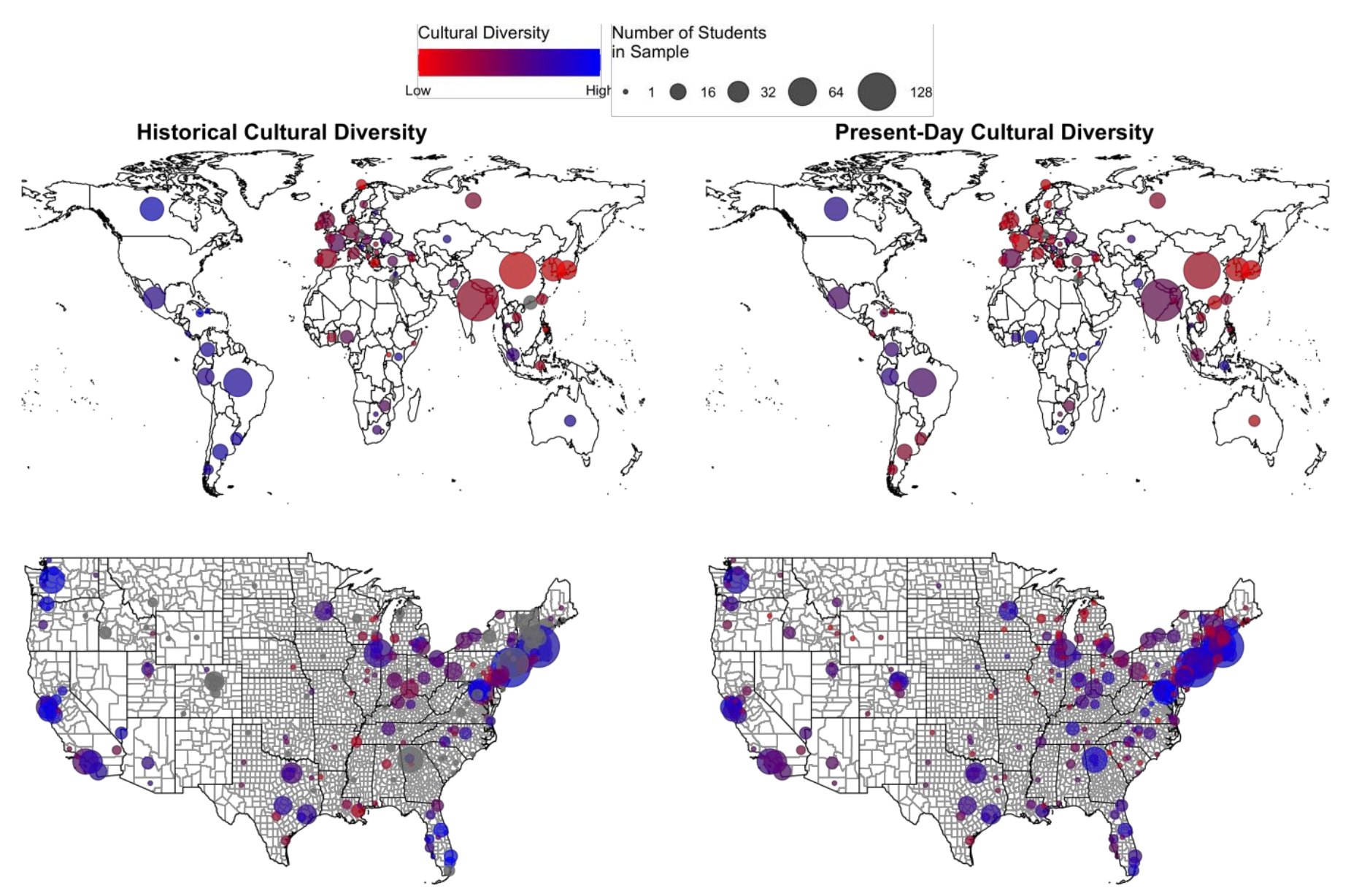

Figure 1. The historical (left side) and present-day (right side) cultural diversity of nations (above) and U.S. counties (below) represented in the sample. Bubble size indicates the number of participants from each geographic region and color indicates higher (blue) or lower (red) cultural diversity. Grey bubbles indicate missing cultural diversity data. Historical cultural diversity of international students' home nations is derived from estimates of historical migration patterns over the last 500 years (Niedenthal et al., 2019), and present-day diversity of their home nations is ethnic fractionalization (Alesina et al., 2003). The historical cultural diversity of the U.S. students' home counties is calculated from self-reported ethnic background from the U.S. Census; present-day diversity comes from a Facebook-based indicator of their hometown county's connectedness to other nations. U.S. students are not visualized in the world map. 
For students who grew up in the U.S., we quantified their home counties' cultural diversity using two indices. To capture long-history demographic trends, we used county-level 2010 census data on self-reported ethnic background to compute regional cultural diversity (Ruggles et al., 2020). We complemented this with a present-day index of U.S. counties' international connectivity based on aggregated 2016 Facebook friendship data made available by the social networking platform (Bailey et al., 2018). We summed the population-adjusted number of Facebook friendships connecting each U.S. county to other nations; this indicator captures both the number and diversity of a U.S. county's international friendships.

We used social brokerage as our primary network outcome variable, given the intuitive connection between exposure to cultural diversity and the ability to connect to a diversity of people in the network. Greater social brokerage is associated with more culturally diverse friends because social networks_-including the present sample—reliably demonstrate homophily. We also, however, considered the cultural diversity of the students' friends.

The central question of the present work is whether the cultural diversity of a person's formative social environment prepares them to create new and diverse social ties. We hypothesized that growing up in a diverse cultural environment predisposes people to becoming social brokers in a new environment because they have greater experience interacting with people unlike themselves. An analysis of individuals who play multiple characters in massively multiplayer online worlds suggested that people are moderately consistent in the positions they occupy in different networks - a broker in one network is likely to become a broker in a new network (Burt, 2012). This suggests that an individual's personality and background can predispose them to brokerage: some people prefer to invest effort into social networking and 
coordinating across groups and are more willing and/or able to be friends with people who are different from them. They may then carry this predisposition with them to a new community.

Our second question was whether and how experience with cultural diversity shapes personality in a way that predisposes one to become a social broker. We therefore explored whether relevant personality traits moderated the effect of cultural diversity on brokerage. Selfmonitoring (Snyder, 1974) is the personality trait that best predicted social brokerage in a metaanalysis of 138 organizational social networks and was therefore our primary personality measure (Fang et al., 2015). Self-monitoring reflects the tendency to attend and adapt to the demands of a social situation. Compared to low self-monitors, high self-monitors tend to be more interpersonally adaptable, adjusting their behavior and expression depending on their current social partner (Friedman \& Miller-Herringer, 1991; Furnham \& Capon, 1983; Wilmot et al., 2016). High self-monitors are also more nonverbally expressive (Renner et al., 2008; Riggio \& Friedman, 1982) and match new acquaintances in their willingness to self-disclose, generating a sense of intimacy (Laux \& Renner, 2002; Shaffer et al., 1982). This social adaptability helps high self-monitors form new friendships, especially with strangers (Sasovova et al., 2010). For example, high self-monitoring Korean immigrants in Canada formed more non-Korean social ties than their low self-monitoring compatriots (Oh \& Kilduff, 2008). The network benefits of high self-monitoring are realized most effectively in people who are also perceived as empathic_ — put another way, social adaptability needs to be perceived as genuine and interested rather than instrumental or anxious (Kleinbaum et al., 2015). Indeed, social connection and career success are related to the degree to which a person demonstrates plasticity and acquisitiveness (acquisitive self-monitoring) rather than instability (protective self-monitoring; Wilmot, Kostal, Stillwell, \& Kosinski, 2017). In addition to self-monitoring, we included the 
following personality dimensions relevant to social adaptability and networking: extraversion, openness to new experiences, ability to work on teams, empathy, teamwork, conflict management, and social influence.

The present sample — a set of large, culturally diverse, and fully-characterized social networks - enables the first ever examination of the impact of a person's cultural background on their capacity to become a social broker. The indicators of cultural diversity complement validated measures based on genetic, archival, and census records with a new estimate of diversity of social ties based on Facebook data. The Facebook social connectivity data, which was only recently made available, provides an unprecedented snapshot of the social ties that connect U.S. counties to the rest of the world. With this unique combination of data sources, we ask how the connectedness of a population influences the future connectedness of its individual members.

\section{Method}

\section{Participants}

Participants were eight entire first-year cohorts from consecutive years of a Masters of Business Administration (MBA) program at a private university in the United States $(\mathrm{N}=2,257)$. Cohorts ranged in size from 267 to 293 students. Of those students, 836 identified as female and 1,418 as male. The sample included 1,461 students with the United States as their primary legal nationality and 773 students with another nation as their primary legal nationality (23 did not report). 1,146 students were White, non-Hispanic; 99 were Hispanic/Latinx; 105 were Black, non-Hispanic; 313 were Asian/Asian American/Pacific Islander; 13 were Native American; 35 multiracial, and 544 did not respond. Students completed two social network surveys as a part of their coursework; the first cohort studied only completed one survey. We collected students' 
hometown information from the MBA student directory, which was originally combined with their survey data for pedagogical reasons within the context of the course. All procedures were completed in accordance with the standards of the Committee for the Protection of Human Subjects, which determined that any data collected for pedagogical purposes could be deidentified and considered as archival data to be used freely. A separate coder deidentified the dataset before its use in the present study. This study was not preregistered.

\section{Social network survey}

Participants completed a first social network survey in September, approximately 5 weeks into the first term of their first year, although one cohort completed the first social network survey in November. All but the first cohort also completed a second social network survey later in the school year, between November and May. Only 6 students failed to complete the first survey, resulting in $99.73 \%$ completion rate; 15 students (excluding the first cohort) failed to complete the second survey, resulting in a $99.24 \%$ completion rate.

Participants received an email linking to an online social network survey that was designed to assess their positions within the first-year MBA community. They were prompted to "Consider the people with whom you like to spend your free time. Since you arrived at [institution name], who are the classmates you have been with most often for informal social activities, such as going out to lunch, dinner, drinks, films, visiting one another's homes, exercising together, and so on?" (adapted from Burt, 1992). To ensure completeness, participants indicated the presence of a social tie by placing a checkmark next to classmates' names, which were presented in alphabetized lists of each of the four student sections of the program.

In the first survey completed in autumn, participants reported an average of 26.4 social ties $($ median $=20, \mathrm{SD}=23.3, \min =1, \max =180)$. For those 7 cohorts that completed a second 
survey in winter or spring, participants reported an average of 38.6 social ties (median $=29$, SD

$=33.0, \min =2, \max =275) .{ }^{1} \mathrm{We}$ then graphed directed and undirected, unweighted social

networks for each cohort. The directed graphs retained information about the direction of

reported friendships (e.g., that Person A says Person B is a friend), while the undirected graphs

consisted of reciprocated friendships only (e.g., a tie will exist between A and B only if they both reported a friendship).

Brokerage is the primary network metric of interest. Social brokers are connected to a diversity of people in the social network - they connect otherwise unconnected subcommunities (Burt, 1992).We used the igraph package for R (Csardi \& Nepusz, 2006) to calculate network constraint for each participant. Constraint $\left(\mathrm{C}_{\mathrm{i}}\right)$ is calculated for each person $i$ in an undirected network using the following equation:

$$
\mathrm{C}_{\mathrm{i}}=\sum_{j=1}^{n}\left(P_{i j}+\sum_{q=1}^{n} P_{i q} P_{q j}\right)^{2}
$$

where $P_{i j}$ is the proportion of person $i$ 's total networking activity that is invested in person $j$; the inner summation captures the indirect connection between person $i$ and person $j$ through each person $q$. For instance, if person $i$ is directly connected to 4 people, including $q$, then $q$ receives $1 / 4$ of $i$ 's networking activity. If person $q$ is, in turn, directly connected to 5 people, including $j$, then $j$ receives $1 / 5$ of $q$ 's output. This is equivalent to $i$ indirectly investing $P_{i q} P_{q j}=1 / 4 \times 1 / 5$, or the equivalent of $1 / 20$ of $i$ 's networking activity, on $j$ through their shared connection with $q . \mathrm{C}_{i}$ is the sum of the squared proportions of direct and indirect network activity person $i$ invests in

\footnotetext{
${ }^{1}$ These relatively high numbers of contacts are consistent with many students' belief that 'networking' is an important goal during business school. Students have ample opportunities to socialize in the first year of the program, as most of them live together in dorms and students eat their meals together, attend classes, and work on team projects. We suspect that the extreme outliers - such as the person citing 180 friends - not only socialize more than others, but also interpreted the question to be more inclusive than other people did.
} 
every other person $j$. In sum, if person $i$ 's network is densely connected, with many contacts who are linked to one another, that network structure is highly constrained and low on brokerage.

To correct for the constraint measure's positive skew and the fact that it is the conceptual inverse of brokerage (high brokerage = low constraint), we reversed and square-rooted it, then standardized the resulting brokerage scores.

\section{Student nationality and hometown}

We collected students' nationality and county information from the registrar, which lists their nation(s) of citizenship, and from the student directory, where students provided their hometown. We used the first place of citizenship listed for students with multiple nationalities. The self-reported hometown information is inherently subjective because students who have lived in multiple places decided which counted as "home" for them. Keeping this limitation in

mind, for U.S. students (students whose primary nationality is U.S.) we determined the county of their hometown to match with the county-level Facebook data and census data.

\section{Personality measures}

Cultural diversity might amplify socially adaptable personality traits (Zimmermann \& Neyer, 2013). We tested this prediction in the present dataset, as participants in some years were asked to complete various personality questionnaires reported in more detail elsewhere (Feiler \& Kleinbaum, 2015). We selected the following self-report personality measures we considered relevant to social adaptability: self-monitoring, extraversion, openness to new experiences, and emotional and social competence. The first three were measured using self-report at the same time as the first social network survey was administered, while self- and peer-report emotional and social competence were measured in the context of a fall term course. See Table 1 for median personality scores for U.S. and international students. 
Self-monitoring. Three cohorts completed Snyder \& Gangestad's (1986) 18-item selfmonitoring scale. Self-monitoring as a construct has long been criticized for its internal validity, with many arguing it captures multiple personality constructs (e.g., Wilmot et al., 2017) The revised scale used here is psychometrically superior to Snyder's (1974) original 25-item scale, but an exploratory factor analysis using the present sample nonetheless suggested a 3-factor

\begin{tabular}{|c|c|c|}
\hline Characteristic & American, $\mathrm{N}=1,461^{1}$ & International, $\mathrm{N}=785^{1}$ \\
\hline Brokerage & $-0.01(-0.57,0.51)$ & $-0.31(-0.84,0.16)$ \\
\hline Indegree nonreciprocity & $0.50(0.33,0.67)$ & $0.52(0.33,0.71)$ \\
\hline Outdegree nonreciprocity & $0.41(0.25,0.56)$ & $0.46(0.28,0.62)$ \\
\hline Number of American friends & $17(8,30)$ & $7(3,14)$ \\
\hline Number of international friends & $3(1,7)$ & $10(4,18)$ \\
\hline Total Friends & $22(11,37)$ & $18(8,33)$ \\
\hline Portion of friends that are American & $0.83(0.73,0.91)$ & $0.44(0.25,0.59)$ \\
\hline Cultural diversity of friends & $31(17,45)$ & $64(50,74)$ \\
\hline ESCI conflict management (self-report) & $0.08(-0.62,0.43)$ & $0.08(-0.62,0.77)$ \\
\hline $\mathrm{ESCl}$ conflict management (peer-report) & $0.23(-0.40,0.87)$ & $-0.30(-0.98,0.37)$ \\
\hline ESCl empathy (self-report) & $0.00(-0.71,0.71)$ & $0.00(-0.35,0.71)$ \\
\hline $\mathrm{ESCl}$ empathy (peer-report) & $0.14(-0.51,0.73)$ & $0.10(-0.58,0.65)$ \\
\hline $\mathrm{ESCl}$ influence (self-report) & $0.04(-0.64,0.72)$ & $0.04(-0.64,0.72)$ \\
\hline $\mathrm{ESCl}$ influence (peer-report) & $0.24(-0.41,0.78)$ & $-0.28(-0.97,0.38)$ \\
\hline ESCI teamwork (self-report) & $0.06(-0.67,0.78)$ & $0.06(-0.67,0.78)$ \\
\hline ESCI teamwork (peer-report) & $0.22(-0.44,0.88)$ & $-0.13(-0.77,0.47)$ \\
\hline Openness to new experiences & $0.04(-0.64,0.72)$ & $0.04(-0.64,0.72)$ \\
\hline Extraversion & $0.23(-0.44,0.83)$ & $-0.10(-0.95,0.40)$ \\
\hline Self-monitoring Factor 1 & $0.52(-0.19,0.52)$ & $-0.19(-0.89,0.52)$ \\
\hline Self-monitoring Factor 2 & $-0.24(-0.81,0.90)$ & $-0.24(-0.81,0.47)$ \\
\hline Self-monitoring Factor 3 & $-0.32(-1.29,0.66)$ & $-0.32(-0.32,0.66)$ \\
\hline
\end{tabular}

Table 1. Median and inner quartile values for network and personality scores, reported separately for U.S. and international students. 
solution (see Online Materials, https://rpubs.com/peerReview1234/cultural-diversity).

Factor 1 (items 1, 9, 14, 15, and 16), which we call social efficacy, captures social selfconfidence and social abilities with statements like, "I am not particularly good at making other people like me" (reversed) and "I feel a bit awkward in public and do not show up quite as well as I should" (reversed). Factor 2 (items 4, 5, 6, 7, 12, and 13), which we call social performativity, captures people's motivation and ability to perform and entertain with statements like, "I can make impromptu speeches even on topics about which I have almost no information" and "I guess I put on a show to impress or entertain others." And Factor 3 (items 8, 10, and 11), which we call social role adoption, captures people's tendency to adjust to the social context with the following items: "In different situations and with different people, I often act like very different persons"; "I'm not always the person I appear to be"; and "I would not change my opinions (or the way I do things) in order to please someone or win their approval" (reversed).

Extraversion and openness to new experiences. We obtained Extraversion and Openness to New Experiences scores for 5 of the 8 cohorts: 2 cohorts completed the 44 item Big 5 Inventory (John et al., 1991), and 3 cohorts completed the 60 item HEXACO Personality Inventory (Ashton \& Lee, 2009). Trait scores on the Big 5 and HEXACO inventories were strongly correlated in prior work (Ashton \& Lee, 2009), so we standardized the Extraversion and Openness scores within-cohort in order to treat scores from different inventories as the same variable.

Emotional and Social Competencies Inventory. All 8 cohorts completed the Hay Group's University Edition of the multi-rater Emotional and Social Competencies Inventory (ESCI; see Kleinbaum et al., 2015). The ESCI comprises peer- and self-report scales related to emotional and social competence. Students completed the ESCI in the fall term of their first year, 
rating themselves and their study group partners on 70 Likert-type items (modal number of peer raters was 5 and ranged from 2 to 5). We selected four ESCI subscales, each comprising 5 items, that we hypothesized to be relevant to social brokerage and diversified social connections: teamwork, empathy, conflict management, and influence. The teamwork subscale includes items like "works well in teams by being supportive" and "works well in teams by being respectful of others"; the empathy subscale included items like "understands others from different backgrounds" and "understands others by listening attentively"; the conflict management subscale included items like "tries to resolve conflicts by finding a solution that addresses everyone's interests" and "resolves conflict by bringing it into the open"; and the influence subscale included items like "convinces others by getting support from key people" and "convinces others by engaging them in discussion." We averaged the peer-reported responses for each student and included the peer- and self-report versions of all four measures in our analyses. The peer- and self-report scores for each subscale are surprisingly uncorrelated, so we did not combine them (teamwork $r=.242$, empathy $r=.171$, conflict management $r=.082$, influence $r$ $=.086)$.

\section{Population-level measures of cultural diversity}

We quantified both historical and present-day cultural diversity of students' home region separately for U.S. and international students, resulting in four primary population-level diversity predictors. To quantify the historical cultural diversity of international students' home nations, we used a nation-level measure of ancestral diversity derived from estimates of historical migration patterns over the last 500 years (Niedenthal et al., 2019). We quantified present-day diversity of their home nations using an index of ethnic fractionalization (Alesina et al., 2003). For students whose primary nationality is the U.S., we quantified their home counties' historical 
cultural diversity using self-reported ethnic background pulled from Census data. And for their indicator of present-day diversity, we used a Facebook-based indicator of their hometown county's connectedness to other nations.

When relevant, we transformed original percentage measures into diversity scores using the Herfindahl-Hirschman Index (HHI; D. Waggoner, 2018). The HHI is a measure of concentration most often used to estimate the extent to which an economic market is monopolized, but here we use it to estimate the extent to which a population is ethnically “monopolized" (i.e., homogeneous). If $100 \%$ of a U.S. county's Census sample reported their ethnic background as Irish, for instance, that county would have an untransformed HHI score of 1, indicating absolute homogeneity; as the county increases in ethnic background diversity, the untransformed HHI score decreases. Diversity was calculated using HHI as follows:

$$
\begin{gathered}
h_{c}=\sum s_{e}^{2} \\
D_{c}=\sqrt{\max (h)-h_{c}}
\end{gathered}
$$

Where $s_{e}$ is the proportion of the population that descends from a particular ethnic/national background $e$ and $h_{c}$ is the untransformed HHI for nation or county $c$. We reversed HHI scores by subtracting them from the maximum HHI, then square-rooted them to correct for skewness to calculate our diversity score $D_{c}$.

Historical diversity of nations. Our measure of nation-level historical diversity comes from estimated patterns of long-history migration (Rychlowska et al., 2015). The World Migration Matrix (WMM; Putterman \& Weil, 2010) estimates the proportion of 165 present-day nations' populations that descend from ancestors from every other nation as of 1500 C.E. From the WMM we computed transformed HHI scores for each nation (Figure 1). China, Norway, Greece, South Korea, and Japan have the lowest scores, indicating near-perfect ancestral 
homogeneity, and Jamaica and Canada have the highest scores. Recent work has used a similar WMM-based metric of ancestral cultural diversity to explain cross-cultural differences in nonverbal behavior (Niedenthal et al., 2019).

Present-day diversity of nations. Alesina et al. (2003) created a measure of ethnic fractionalization for 190 nations and 650 distinct ethnic groups by compiling a variety of estimates from the 1990s. Ancestral diversity is an estimate of migration from one geographic region to another over 500 years, while ethnic fractionalization is an estimate of present-day ethnic diversity, which might be due to historical or contemporary migration, but may also be due to newly-emerged ethnic identities (e.g., the Tutsis and the Hutus in Rwanda). Alesina et al. (2003) estimated ethnic fractionalization scores as reversed HHI proportions that range from 0 (e.g., United Arab Emirates) to .93 (e.g. Uganda), with higher scores indicating more present-day diversity. The present-day and historical cultural diversity of the nations represented in our sample are somewhat positively correlated $(r=.267)$.

Historical diversity of U.S. counties. We calculated historical diversity for each U.S. county using Census data reported in the IPUMS dataset (Ruggles et al., 2020). The dataset includes self-reported ethnic ancestry from over a million people surveyed in 2016, along with their U.S. county of residence. From this, we calculated transformed HHI scores for each county, with higher scores indicating greater historical diversity. The least historically diverse county represented in our student sample is Outagamie County, Wisconsin (.024) and the most historically diverse county is Montgomery County, Maryland (.049), a suburb of Washington, DC.

Present-day diversity of U.S. counties. For present-day diversity of U.S. counties, we computed the diversity of each county's international connectedness via social media. Facebook 
researchers captured a snapshot of all friendships of active Facebook users at a moment in time in 2016 (Bailey et al., 2018). They estimated users' location from their information and activity on Facebook, including the city listed in their profiles and information gathered from their devices. Using data from all Facebook users living in each U.S. county, they computed a relative measure of the number of Facebook friendships connecting people in each U.S. county to people in 157 other nations as of 2016 (note they only used nations with a population greater than 1 million; Bailey et al., 2018). For each nation, they normalized the number of friendships connecting it to each U.S. county, so the county with the greatest number of friendships received a score of 1,000,000 (this adjusts for nation-level variability in overall population, total number of Facebook users, and total number of Facebook users with friends in the U.S.). To correct for U.S. county population, they computed the relative probability of friendship as $(100 \times$ Normalized_Number_of_Friendships) / Population_of_county.

We could not use the HHI approach we used for the other diversity indicators because of the way the county-level friendship counts were normalized. Therefore, in order to create a summary statistic at the U.S. county level that indicates the degree to which its residents are exposed to cultural diversity, for each county we added together the normalized number of friendships with all nations, then log-transformed the scores to correct for skew (a square-root transformation did not sufficiently correct the skew). By this metric, in our student sample the least internationally-connected county is Somerset County, Pennsylvania (12.72) and the District of Columbia is the most internationally-connected county (81.33; see Figure 1). The historicaland present-day cultural diversity metrics for the U.S. counties in our sample are moderately positively correlated $(r=.480)$. 


\section{Results}

Network graphs based on the eight cohorts' first survey appear in Figure 2. In the first section we report models predicting social brokerage at the time of the first survey as a function of 1) historical and present-day cultural diversity of international students' home nations and 2)

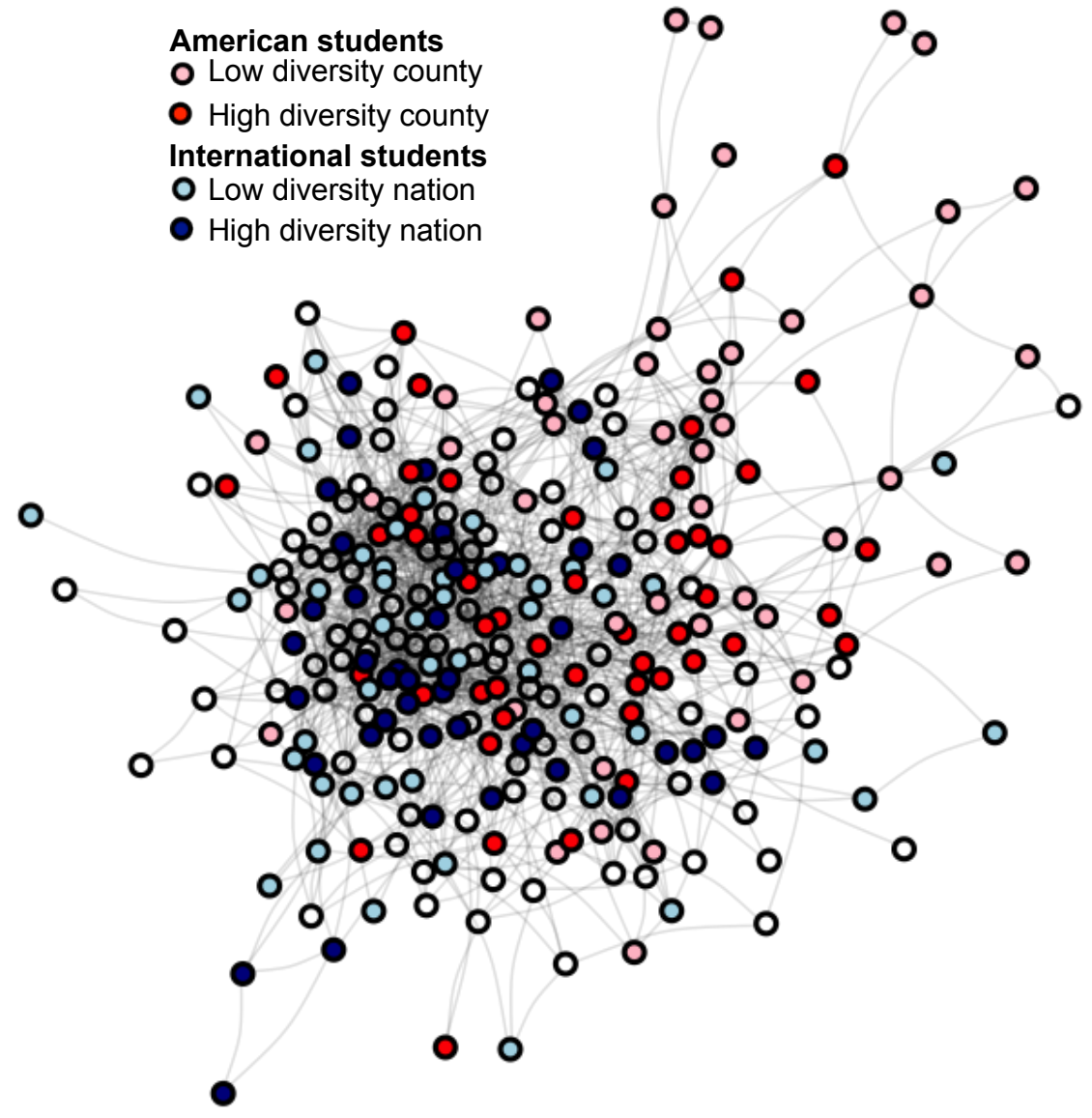

Figure 2. The social network graph of one cohort. Edges represent reciprocated friendships. Light blue and dark blue nodes are students from U.S. counties below and above the median in historical diversity, respectively. Light and dark red nodes are international students from nations with below or above the median in historical diversity. Node position is determined using the Fruchterman-Reingold force-directed algorithm, which pulls highly connected nodes to the center (Fruchterman \& Reingold, 1991). 
historical and present-day cultural diversity of U.S. students' home counties. Then, for any effects with credibility intervals that excluded 0 at time 1 , we ran additional analyses on both waves of data including time as a moderator. Although we explored potential effects of cultural diversity on other network measures - the diversity of students' friends and the reciprocity of their friendships — none of these were predicted by cultural diversity. See Online Materials for all model estimates (https://rpubs.com/peerReview1234/798393).

In the second section, we report the results from models predicting students' socially adaptive personality trait scores from their home region's cultural diversity. See Online Materials for additional results, including models controlling for total number of friends, race, several culture-level covariates (e.g., the English-speaking status, relational mobility, and normative tightness of students' home nations). While we do not have permission to share student-level data publicly, the population-level variables are available online (https://osf.io/ycpwt/).

\section{Bayesian regression model specification}

The first set of models was estimated with Bayesian multilevel linear regression using Markov Chain Monte Carlo in R (R Development Core Team, 2008). We opted for a Bayesian model fitting approach to avoid convergence issues often encountered with mixed-effects models fit with restricted maximum likelihood (REML). When we included by-nation and by-county random intercepts, initial models computed using Restricted Maximum Likelihood in the lme4 R package (Bates, Mächler, Bolker, \& Walker, 2015) failed to converge, possibly because of the sparsity of the data at the group level $-47 \%$ of U.S. counties and $34 \%$ of nations are represented by a single student. We therefore fit the models using the stan_lmer() function in the rstanarm $\mathrm{R}$ package (Gabry \& Goodrich, 2016), which uses the STAN probabilistic programming language 
(see Online Materials for models alternatively fit with REML, ordinary least squares, and cluster-robust variance estimators).

For each regression model, rstanarm runs four randomly-initialized chains for 2,000 iterations (the first 1,000 are discarded as warmup draws) and checks for convergence by computing the ratio of the within-chain variance to the between-chain variance, which is close to 1 when a model successfully converges. We used weak, uninformative priors for all model coefficients since we did not have evidence-based predictions about the size or direction of any effects: our normal prior distributions $(M=0, S D=3)$ encompassed all plausible coefficients. We concluded there was evidence for an effect whenever the $95 \%$ credibility interval excluded zero and we report mean coefficients $(\beta)$, Monte Carlo Standard Errors (MCSE), standard deviation of the estimates (SD), 95\% credibility intervals (95\% CI), effective sample sizes $\left(\mathrm{N}_{\text {eff }}\right)$, and a measure of whether the Monte Carlo chains were at equilibrium ( $\hat{r}$, with values near 1 indicating equilibrium).

\section{Cultural diversity predictors of social brokerage}

\section{Nation-level historical cultural diversity predicts international students'social}

brokerage. We first regressed brokerage scores (Z-scaled for interpretability) of international students on the historical cultural diversity of their home nations (based on migration patterns) with random intercepts by nation (see Figure 1 for map of historical diversity; see top left graph in Figure 3 for scatterplot). As hypothesized, nation-level historical diversity positively predicted the brokerage scores of international students (see Table 2 for model estimates). Brokerage for a student from a nation with the lowest possible historical diversity score (e.g., China, Norway, Greece) is predicted to be .493 standard deviations ${ }^{2}$ lower than for a student

\footnotetext{
${ }^{2}$ Standard deviations are calculated for the entire sample including international and U.S. students.
} 
from the nation with the highest historical diversity score (Jamaica).

We then modeled brokerage data from both social network survey waves and added fixed effects for time (first vs. second survey) and its interaction with historical diversity (and bysubject random intercepts). The interaction term indicated that the effect of historical diversity on brokerage did not change over the course of the academic year $(\beta=0.105$, MCSE $=$ $\left.0.003, \mathrm{SD}=0.214,95 \% \mathrm{CI}=[-0.295,0.559], \mathrm{N}_{\mathrm{eff}}=4,700, \hat{\mathrm{r}}=1.001\right)$

\begin{tabular}{|c|c|c|c|c|c|c|c|c|}
\hline $\begin{array}{l}\text { Cultural Diversity } \\
\text { Measure }\end{array}$ & Parameter & $\boldsymbol{\beta}$ & MCSE & SD & $\begin{array}{l}2.5 \% \\
\text { C.I. }\end{array}$ & $\begin{array}{l}97.5 \% \\
\text { C.I. }\end{array}$ & $\mathbf{N}_{\text {eff }}$ & $\hat{\mathbf{r}}$ \\
\hline \multicolumn{9}{|l|}{$\begin{array}{l}\text { International } \\
\text { students }\end{array}$} \\
\hline \multirow[t]{2}{*}{ historical diversity } & Intercept & -0.302 & 0.001 & 0.05 & -0.401 & -0.202 & 1921 & 1.001 \\
\hline & Slope & 0.139 & 0.001 & 0.051 & 0.040 & 0.240 & 1676 & 1.004 \\
\hline \multirow[t]{2}{*}{ present-day diversity } & Intercept & -0.265 & 0.001 & 0.05 & -0.360 & -0.163 & 1418 & 1.003 \\
\hline & Slope & 0.107 & 0.001 & 0.044 & 0.022 & 0.193 & 1758 & 1 \\
\hline \multirow[t]{2}{*}{$\begin{array}{l}\text { U.S. students } \\
\text { historical diversity }\end{array}$} & Intercept & -0.011 & 0 & 0.029 & -0.071 & 0.046 & 6465 & 0.999 \\
\hline & Slope & 0.068 & 0 & 0.028 & 0.012 & 0.125 & 6488 & 1 \\
\hline \multirow[t]{2}{*}{ present-day diversity } & Intercept & -0.023 & 0 & 0.025 & -0.071 & 0.025 & 6522 & 0.999 \\
\hline & Slope & 0.047 & 0 & 0.024 & -0.001 & 0.094 & 7325 & 0.999 \\
\hline
\end{tabular}

Table 2. Model estimates from multilevel Bayesian models predicting standardized network brokerage from the four measures of cultural diversity, separately for international and U.S. students. Credibility intervals for slopes that exclude 0 are in bold. 

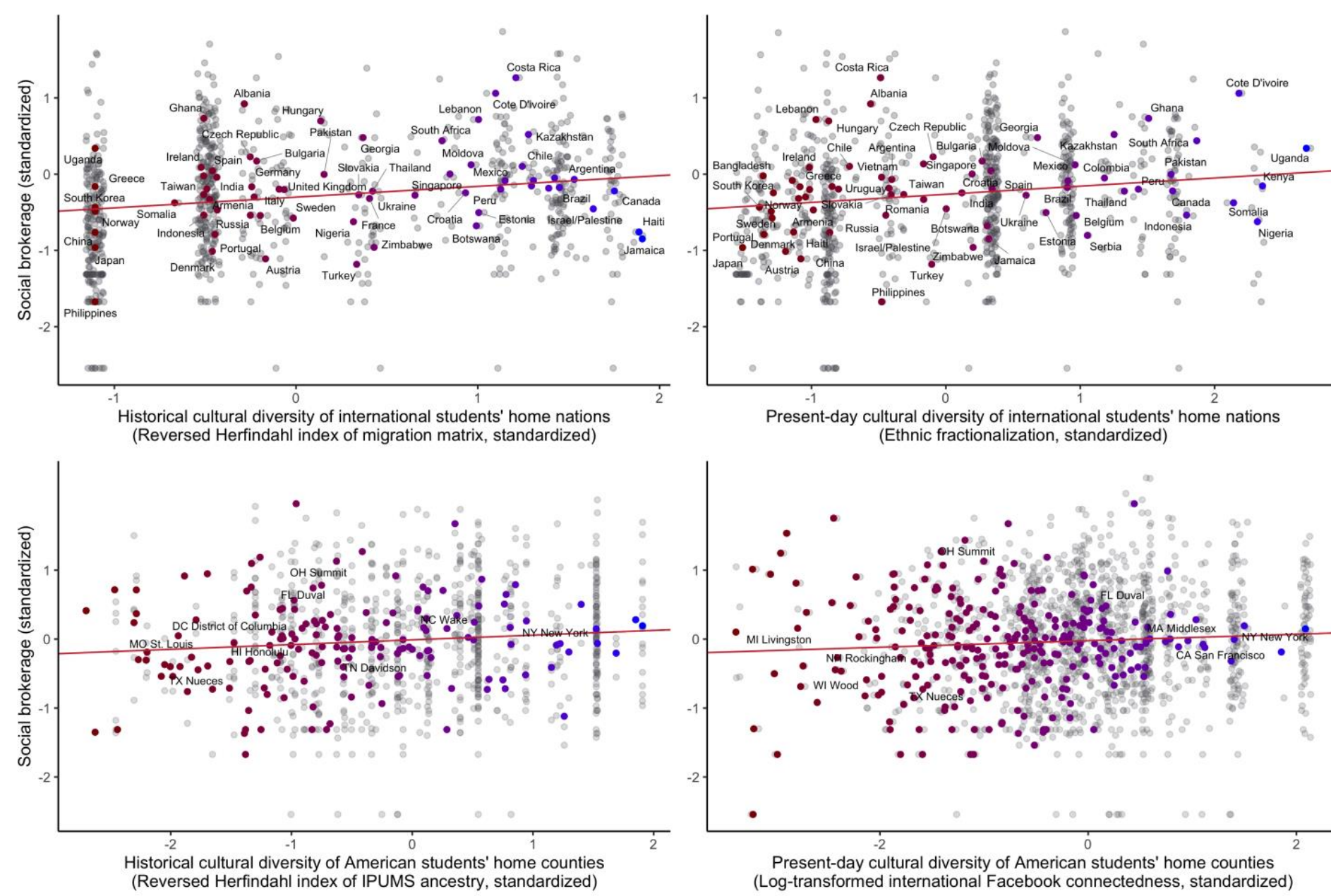

Present-day cultural diversity of American students' home counties (Log-transformed international Facebook connectedness, standardized)

Figure 3. Predicting international (above) and U.S. (below) students' brokerage scores at time 1 from the historical and present-day cultural diversity of their home communities. Red lines are the estimated fixed effects from a Bayesian multilevel model. Grey points are students' scores and red-blue points are the average scores for each nation or county (bluer for higher cultural diversity). Students from more diverse home communities tend to be higher in network brokerage in their MBA cohorts, indicating more diversified social ties. The only slope estimate with a credibility interval that includes 0 , indicating a lack of an effect, is present-day cultural diversity of U.S. students (bottom right).

\section{Nation-level present-day cultural diversity predicts international students' social}

brokerage. We repeated the above steps using present-day diversity as the predictor (Alesina et al., 2003). Again supporting the prediction that cultural diversity leads to brokerage, international students from the nation lowest on present-day diversity (Japan) were predicted to have 
brokerage scores .449 standard deviations lower than students from the nation highest on present-day diversity (Uganda, see Table 2 and Figure 3). The time interaction model indicated the effect of present-day diversity on brokerage did not change from time 1 to time $2, \beta=0.116$, $\operatorname{MCSE}=0.003, \mathrm{SD}=0.220,95 \% \mathrm{CI}=[-0.314,0.580], \mathrm{N}_{\mathrm{eff}}=4,298, \hat{\mathrm{r}}=1.000$.

\section{County-level historical diversity predicts U.S. students' social brokerage. Next we}

predicted the brokerage scores of U.S. students from the historical diversity of their home counties (Ruggles et al., 2020) with random intercepts for U.S. counties. Once again, and at a different historical and geographic scale, historical cultural diversity positively predicted the brokerage scores of students (see Table 2 and Figure 3). U.S. students from the county lowest in historical diversity (Outagamie County, Wisconsin) were predicted to have a brokerage score .313 standard deviations lower than students from the U.S. county highest in historical diversity (Montgomery County, Maryland). And again, the effect of diversity did not change over the course of the academic year, $\beta=0.229, \mathrm{MCSE}=0.009, \mathrm{SD}=0.430,95 \% \mathrm{CI}=[-0.457,1.280]$, $\mathrm{N}_{\text {eff }}=2,085, \hat{\mathrm{r}}=1.000$.

\section{County-level present-day cultural diversity did not predict U.S. students' social}

brokerage. We repeated the above steps for U.S. students' present-day diversity, based on international Facebook friendship patterns, as the focal predictor variable (Bailey et al., 2018). The effect for present-day U.S. county-level cultural diversity was in the expected positive direction but its 95\% credibility interval did not exclude 0 (see Table 2 and Figure 3).

To summarize the findings for brokerage, we showed that people from culturally diverse geographic regions are more likely to become brokers in a new social network. International students from nations with a more extensive history of immigration and with more present-day ethnic fractionalization were higher in brokerage in the MBA friendship network than 
international students from more homogeneous nations. Likewise, students from U.S. counties with more diverse ethnic backgrounds were higher in brokerage than U.S. students from less historically diverse counties. However, the diversity of U.S. counties' present-day ties did not substantially predict students' brokerage.

\section{Cultural diversity predictors of socially adaptive personality traits}

Using the same modeling approach, we used our four nation and U.S. county measures of cultural diversity to predict our socially adaptive personality measures - the three selfmonitoring factors, extraversion, openness to new experiences, and peer- and self-reported teamwork, empathy, conflict management, and influence. For brevity's sake, we report only the estimates with credibility intervals that exclude zero, but see the Online Materials for all model estimates. The effective sample sizes for these models are smaller than our original sample size because some cohorts did not complete the personality measures.

Personality variability. Neither of the U.S. county-level diversity measures predicted any of the personality variables of interest in the U.S. students. However, the historical diversity of international students' home nations positively predicted their Z-scored self-reported extraversion, $\beta=0.261, \mathrm{MCSE}=0.001, \mathrm{SD}=0.092,95 \% \mathrm{CI}=[0.086,0.444], \mathrm{N}_{\text {eff }}=4,087, \hat{\mathrm{r}}=$ 1.000. A student from the least historically diverse nation (China, Norway, Greece) is predicted to be .786 standard deviations lower on extraversion than a student from the most historically diverse nation (Jamaica).

Unexpectedly, historical diversity negatively predicted the social role adoption factor of self-monitoring, $\beta=-0.188, \mathrm{MCSE}=0.001, \mathrm{SD}=0.065,95 \% \mathrm{CI}=[-0.315,-0.058], \mathrm{N}_{\text {eff }}=$ 4,322, $\hat{\mathrm{r}}=1.000$. A student from a country with the lowest ancestral diversity in our sample (e.g., China, Norway, or Greece), is predicted to be .568 standard deviations higher on the social role 
adoption factor of self-monitoring compared to a student from a country with the highest ancestral diversity (Jamaica). This suggests, counter to our expectations, that at least one aspect of self-monitoring — namely, the tendency to "not always [be] the person [they] appear to be"-is higher in less historically diverse nations.

The social role adoption factor of self-monitoring was the only personality measure predicted by nation-level present-day diversity, and again, the relationship was unexpectedly negative, $\beta=-0.185, \mathrm{MCSE}=0.001, \mathrm{SD}=0.062,95 \% \mathrm{CI}=[-0.305,-0.064], \mathrm{N}_{\mathrm{eff}}=4,196, \hat{\mathrm{r}}=$ 1.000. A student from the country lowest in ethnic fractionalization (Japan) is predicted to be .784 standard deviations higher in social role adoption than a student from the country highest in ethnic fractionalization (Uganda).

\section{Does personality moderate the effect of cultural diversity on brokerage? Since}

extraversion and the social role adoption subscale of self-monitoring were predicted by cultural diversity in international students, we explored whether either of these variables moderated the effects of diversity on brokerage. For each of the three personality effects (historical diversity predicting extraversion and social role adoption; present-day diversity predicting social role adoption), in three separate models we regressed brokerage on the personality variable, the diversity variable, and their interaction. None of the interaction terms excluded zero, indicating the effect of cultural diversity on brokerage was not moderated by students' extraversion or social role monitoring (see Online Materials for model estimates).

\section{Discussion}

Here we examined whether people who have grown up in cultures with greater cultural diversity were more likely to become social brokers in a new and culturally diverse social network compared to people from populations with less cultural diversity. We examined a large 
number of people (2,257 first-year MBA students) who collectively formed eight new social networks. The sample was culturally diverse (35\% international students) yet homogeneous on a number of potentially confounding factors (such as education level, age, and presumed motivation to network with peers). The complete sociocentric social network data for the newlyformed community served as a quasi-experimental test of the effect of cultural background on social connectedness.

We predicted social brokerage in the network using geography-based estimates of the cultural diversity of students' home populations, at the national level for international students and the county level for students from the U.S. Both long- and short-term shifts in cultural diversity appear to create environments that prepare the population for forming new and broad social ties. The cultural diversity of international students' home nations, quantified in terms of long-history migration patterns and present-day ethnic fractionalization, predicted the extent to which students acted as social brokers in the network. The same relationship emerged when we measured population cultural diversity at a more granular scale for U.S. counties. The ancestral diversity of a U.S. county, calculated from self-reported ethnic heritage data gathered by the U.S. Census, predicted the brokerage of students in the MBA network. However, the present-day international connectedness of U.S. counties, which we estimated from aggregated Facebook friendship data provided by the social media company (Bailey et al., 2018), did not reliably predict brokerage (although the slope was in the expected direction). The effect of cultural diversity on social network brokerage was moderate: students from the least and most culturally diverse home communities differed in social brokerage by approximately half a standard deviation. Crucially, there was more variability in social brokerage within students from a given 
community than between communities. The present work therefore does not suggest that a person's cultural background inevitably determines their social connectedness.

One possible driver of our effects is simply the sheer number of social ties: as reported in our Online Materials, social brokerage was positively correlated with total number of ties $(r=$ .78). It is possible that students with more friends are more likely to bridge otherwise disconnected parts of the community purely as a result of this increased number of connections. We therefore ran supplementary models predicting brokerage from our cultural diversity variables while controlling for total number of ties. After controlling for total number of ties, the effects of cultural diversity on brokerage persisted for international students but not for the U.S. students.

The effects of cultural diversity on brokerage were larger for international students than for students from the U.S. and, for the latter, only our historical indicator predicted brokerage. There are several possible explanations for these findings. The first is that within-U.S. variability in cultural diversity is smaller than between-nation variability. Even the most cultural homogeneous counties in the U.S. were populated by waves of immigration and are embedded in a larger culture born from dozens of contributing cultures. A second possibility is that international virtual (Facebook) ties may not demand the same level of social adaptability as high levels of in-person, within-population ethnic fractionalization. We cannot expect online exposure to cultural diversity to replicate the effect of living in a culturally diverse neighborhood. A third possibility is that all the U.S. students - even those from culturally homogeneous countiesbenefited socially from being majority group members in the MBA program. Even if a U.S. student actively avoided forming ties with culturally diverse peers, they could still be a broker between other U.S. students, who comprised $65 \%$ of the cohorts. For an international student to 
become a social broker, on the other hand, required crossing cultural and national boundaries because they were minority group members. Experience with culturally diverse friendships could have been more essential, therefore, for international compared to domestic students.

The present work converges with a recent finding that East Asian law school students have more social network homophily_befriending mostly other East Asian students and thereby restricting their networks - compared to South Asian students (Lu, 2021). South Asia is relatively more culturally diverse, by our metrics, than East Asia. For instance, India is .7 standard deviations higher than China on ancestral cultural diversity and 1 standard deviation higher than China on present day cultural diversity. Our results suggest that East Asian students in a U.S. law school and, in the present work, an MBA program, are less prepared than South Asian students to form wide network ties in a culturally diverse context because of their relatively less culturally diverse home populations.

What are the implications of population-level cultural diversity for the formation of social ties? The present work suggests that some international students in U.S. universities might be particularly vulnerable to marginalization and its downstream effects on well-being and academic performance (Rivas et al., 2019). Culturally-aware training for new international students might focus on concrete strategies for, and the benefits of, forming cross-cultural friendships thereby increasing the efficacy of "networking" programs that are often deemed ineffectual or aversive (Casciaro et al., 2014; Kuwabara et al., 2018). If future research finds similar effects of cultural background on social connectedness for non-student populations, similar interventions might improve their outcomes.

Another implication of the present work is that encounters with cultural diversity may provide enduring benefits to the individual ( $\mathrm{Lu}$ et al., 2021) and the benefits of multiculturalism 
can linger over generations within a population (Niedenthal et al., 2019). Our finding is complemented by recent evidence that immigrants from more relationally mobile nations are less lonely (Heu et al., 2020). Nation-level relational mobility-how unstable and malleable a nation's residents perceive their social ties to be-is positively correlated with cultural diversity. We therefore speculate that one way culturally diverse communities prepare people to become social brokers is by exposing them to unstable social networks that provide additional opportunities to make new friends. And by becoming social brokers in their new and diverse environment, they help the entire community reap the social capital benefits of diversity (Laurence, 2011).

The present work does not imply that cultural diversity alone determines one's position in a social network. Here we simply show that a person's prior exposure to diversity is one influential factor. Other constraints of a socio-ecology, such as the spatial proximity of subcommunities (Herdağdelen et al., 2016), also help determine whether a person can form diverse ties and become a broker. In some socio-ecologies, such as those with low residential mobility, having many broad social ties is maladaptive (Oishi \& Kesebir, 2012). And of course, discrimination towards members of a particular culture, between-group disparities, and language barriers will also influence an individual's social ties.

\section{Does cultural diversity shape personality?}

What mechanisms might underlie the link between social connectivity and populationlevel variables like ancestral diversity? Any distal effect must be mediated via proximal behaviors or traits. We explored the role of several adaptable personality traits, including selfmonitoring, which is known to predict social flexibility (Sasovova et al., 2010) and might be fostered by repeated cooperative interactions with diverse others. Prior work suggested that 
ancestral diversity predicts population-level personality traits such as openness to new experiences (Shrira et al., 2018). Unexpectedly, however, the only personality traits predicted by cultural diversity in the present sample were extraversion and the subfactor of self-monitoring we refer to as "social role adoption," or the tendency to hide one's true feelings and conform to the social context. Exploratory moderation analyses did not indicate that the effect of diversity on brokerage depended on either of these dimensions, but this exploratory analysis was limited by sample size. Not all cohorts in the present study were asked to complete personality measures (the social role monitoring subsample $n=286$; the extraversion subsample $n=196$ ). Both extraversion and the social role adoption subfactor of self-monitoring are therefore still worth examining in the future as possible fingerprints of exposure to cultural diversity. But we may find, as have others, that self-monitoring is an unreliable cross-cultural variable (Lukaszewski et al., 2017; Smaldino et al., 2018). The construct and measurement validity of self-monitoring is debated, and future work should employ a revised version of a self-monitoring scale (Lennox \& Wolfe, 1984).

We posit that the effect of cultural diversity on brokerage reflects the accumulation of many social adaptations, such as nonverbal expressivity and social tolerance (Niedenthal et al., 2019). Individual differences in both motivation and ability to socialize with a diversity of people should predict social network outcomes, and both may be shaped by culture. For instance, people from more culturally diverse populations may have internalized norms that make them more willing to make friends with people of diverse backgrounds, even if they have had limited direct exposure to diversity. They may also have more practice with forming new social ties (Heu et al., 2020), as culturally diverse communities tend to experience more frequent social tie formation 
and dissolution (Thomson et al., 2018). Additional research is needed to consider other potential mediators and moderators of the effect of cultural diversity on network position.

\section{Future directions}

Future work should examine the influence of cultural diversity on brokerage in different samples. The international MBA students may be nonrepresentative members of their home nations - after all, they chose to study abroad and may embody the "frontier" personality (Kitayama et al., 2006). If anything, however, this will have generated greater between-culture similarity and led to an underestimation of the effect of cultural differences on social connection. Future work should also examine social networks in a low-diversity host community (e.g., a business school in a nation like Norway). Do people still benefit from prior exposure to cultural diversity regardless of the diversity of their current social environment? Or is this a matter of person-culture fit, such that people with culturally diverse backgrounds are successful specifically in culturally diverse settings (Gebauer et al., 2020; Geeraert et al., 2019)?

It is also important to clarify the social behavioral patterns of social brokers with culturally diverse backgrounds. Are they social chameleons who match the behavioral norms of each social partner? Or are they "authentically" themselves across partners? We speculate the latter. International students from ancestrally diverse nations scored lower on the social role adoption facet of self-monitoring, agreeing less with statements such as, "In different situations and with different people, I often act like very different persons." However, this relationship warrants replication, since social role adoption did not moderate the effect of cultural diversity on brokerage (perhaps given the small subsample of students who completed the self-monitoring scale). People exposed to cultural diversity may become brokers not by changing their behavior, but by seeing the value in cultural differences (Miville et al., 1999). 
We recognize that cultural diversity and the way it was operationalized here is only one way to think about diversity. Other forms of diversity include ethnic/racial, religious, personality, linguistic, political, and skillset diversity which intersect with, but are also separable from, cultural diversity. How various forms of diversity impact social connectedness is an important topic for future research, especially considering the modest effects of our chosen diversity dimensions on brokerage.

Cultural diversity is also important to consider across time. We hope that our approach here highlights the utility of defining cultural diversity not only through a present-day snapshot, but also by taking into account deeper historical contexts. The story of our species is one of migration and cultural and genetic exchange, extending as far back as the intermingling of modern humans with other hominins (Zaidner et al., 2021). In this study we have made an initial attempt to consider the consequences of cultural diversity not only across space, but through time as well. We hope that future research will further broaden and refine how cultural diversity is understood and measured.

Experimentally manipulating the structure of social networks in the lab (Momennejad et al., 2019) will also help to determine the causal direction of the relationship between prior exposure to cultural diversity and social brokerage. Just as cultural diversity predicts network position, the ways in which members of a network are connected can either amplify or homogenize network diversity. Network simulations suggest sparsely-connected social networks that rely on social brokers to transmit information are more likely to maintain cultural diversity and produce more diverse solutions to problems (Derex \& Boyd, 2016; Smolla \& Akçay, 2019).

In summary, we demonstrate that whether an individual forms diverse ties in a social network is predicted, in part, by the diversity of their home community. International students 
from more culturally diverse nations and U.S. students from more culturally diverse counties were more likely to become the bridges that tied diverse networks together. On a global scale, culturally diverse environments broker the exchange of people and resources and cultivate people who, on a local scale, broker the social ties in their communities. 


\section{References}

Alesina, A., Devleeschauwer, A., Easterly, W., Kurlat, S., \& Wacziarg, R. (2003).

Fractionalization. Journal of Economic Growth, 8(2), 40. https://doi.org/10.3386/w9411

Ashton, M. C., \& Lee, K. (2009). The HEXACO-60: A Short Measure of the Major Dimensions of Personality. Journal of Personality Assessment, 91(4), 340-345.

https://doi.org/10.1080/00223890902935878

Bailey, M., Cao, R., Kuchler, T., Stroebel, J., \& Wong, A. (2018). Social Connectedness:

Measurement, Determinants, and Effects. Journal of Economic Perspectives, 32(3), 259280. https://doi.org/10.1257/jep.32.3.259

Bates, D., Mächler, M., Bolker, B., \& Walker, S. (2015). Fitting Linear Mixed-Effects Models Using lme4. Journal of Statistical Software, 67(1), 1-48.

https://doi.org/10.18637/jss.v067.i01

Burt, R. (1992). Structural holes: The social structure of competition (Harvard, MA, Harvard University Press).

Burt, R. S. (2004). Structural Holes and Good Ideas. American Journal of Sociology, 110(2), 349-399. https://doi.org/10.1086/421787

Burt, R. S. (2012). Network-Related Personality and the Agency Question: Multirole Evidence from a Virtual World. American Journal of Sociology, 118(3), 543-591. https://doi.org/10.1086/667856

Burt, R. S. (2019). Network Disadvantaged Entrepreneurs: Density, Hierarchy, and Success in China and the West. Entrepreneurship Theory and Practice, 43(1), 19-50. https://doi.org/10.1177/1042258718783514 
Carnovale, S., \& Yeniyurt, S. (2015). The Role of Ego Network Structure in Facilitating Ego Network Innovations. Journal of Supply Chain Management, 51(2), 22-46. https://doi.org/10.1111/jscm.12075

Casciaro, T., Gino, F., \& Kouchaki, M. (2014). The Contaminating Effects of Building Instrumental Ties: How Networking Can Make Us Feel Dirty. SSRN Electronic Journal. https://doi.org/10.2139/ssrn.2430174

Cattell, V. (2001). Poor people, poor places, and poor health: The mediating role of social networks and social capital. Social Science \& Medicine, 52(10), 1501-1516. https://doi.org/10.1016/S0277-9536(00)00259-8

Chua, R. Y. J., Roth, Y., \& Lemoine, J.-F. (2015). The Impact of Culture on Creativity: How Cultural Tightness and Cultural Distance Affect Global Innovation Crowdsourcing Work. Administrative Science Quarterly, 60(2), 189-227. https://doi.org/10.1177/0001839214563595

Csardi, G., \& Nepusz, T. (2006). The igraph software package for complex network research. InterJournal, Complex Systems, 1695(5), 1-9.

D. Waggoner, P. (2018). The hhi Package: Streamlined Calculation and Visualization of Herfindahl-Hirschman Index Scores. Journal of Open Source Software, 3(28), 828. https://doi.org/10.21105/joss.00828

Derex, M., \& Boyd, R. (2016). Partial connectivity increases cultural accumulation within groups. Proceedings of the National Academy of Sciences, 113(11), 2982-2987. https://doi.org/10.1073/pnas. 1518798113

Eagle, N., Macy, M., \& Claxton, R. (2010). Network Diversity and Economic Development. Science, 328(5981), 1029-1031. https://doi.org/10.1126/science.1186605 
Erickson, B. (2003). Social Networks: The Value of Variety. Contexts, 2(1), 25-31. https://doi.org/10.1525/ctx.2003.2.1.25

Fang, R., Landis, B., Zhang, Z., Anderson, M. H., Shaw, J. D., \& Kilduff, M. (2015). Integrating Personality and Social Networks: A Meta-Analysis of Personality, Network Position, and Work Outcomes in Organizations. Organization Science, 26(4), 1243-1260. https://doi.org/10.1287/orsc.2015.0972

Feiler, D. C., \& Kleinbaum, A. M. (2015). Popularity, similarity, and the network extraversion bias. Psychological Science, 26(5), 593-603.

Friedman, H. S., \& Miller-Herringer, T. (1991). Nonverbal display of emotion in public and in private: Self-monitoring, personality, and expressive cues. Journal of Personality and Social Psychology, 61(5), 766.

Fruchterman, T. M., \& Reingold, E. M. (1991). Graph drawing by force-directed placement. Software: Practice and Experience, 21(11), 1129-1164.

Furnham, A., \& Capon, M. (1983). Social skills and self-monitoring processes. Personality and Individual Differences, 4(2), 171-178.

Gabry, J., \& Goodrich, B. (2016). rstanarm: Bayesian applied regression modeling via Stan. $R$ Package Version, 2(1).

Gebauer, J. E., Eck, J., Entringer, T. M., Bleidorn, W., Rentfrow, P. J., Potter, J., \& Gosling, S. D. (2020). The Well-Being Benefits of Person-Culture Match Are Contingent on Basic Personality Traits. Psychological Science, 31(10), 1283-1293. https://doi.org/10.1177/0956797620951115 
Geeraert, N., Li, R., Ward, C., Gelfand, M., \& Demes, K. A. (2019). A Tight Spot: How Personality Moderates the Impact of Social Norms on Sojourner Adaptation. Psychological Science, 30(3), 333-342. https://doi.org/10.1177/0956797618815488

Gelfand, M. J., Harrington, J. R., \& Jackson, J. C. (2017). The Strength of Social Norms Across Human Groups. Perspectives on Psychological Science, 12(5), 800-809. https://doi.org/10.1177/1745691617708631

Gelfand, M. J., Raver, J. L., Nishii, L., Leslie, L. M., Lun, J., Lim, B. C., Duan, L., Almaliach, A., Ang, S., Arnadottir, J., Aycan, Z., Boehnke, K., Boski, P., Cabecinhas, R., Chan, D., Chhokar, J., D’Amato, A., Ferrer, M., Fischlmayr, I. C., ... Yamaguchi, S. (2011). Differences Between Tight and Loose Cultures: A 33-Nation Study. Science, 332(6033), $1100-1104$.

Girard, J. M., \& McDuff, D. (2017). Historical heterogeneity predicts smiling: Evidence from large-scale observational analyses. Automatic Face \& Gesture Recognition (FG 2017), 2017 12th IEEE International Conference On, 719-726.

Herdağdelen, A., State, B., Adamic, L., \& Mason, W. (2016). The Social Ties of Immigrant Communities in the United States. Proceedings of the 8th ACM Conference on Web Science, 78-84. https://doi.org/10.1145/2908131.2908163

Heu, L. C., van Zomeren, M., \& Hansen, N. (2020). Far away from home and (not) lonely: Relational mobility in migrants' heritage culture as a potential protection from loneliness. International Journal of Intercultural Relations, 77, 140-150. https://doi.org/10.1016/j.ijintre1.2020.05.005 
Hong, Y.-Y., Morris, M. W., Chiu, C., \& Benet-Martínez, V. (2000). Multicultural minds: A dynamic constructivist approach to culture and cognition. American Psychologist, 55(7), $709-720$.

John, O. P., Donahue, E. M., \& Kentle, R. L. (1991). Big five inventory. Journal of Personality and Social Psychology.

Jokela, M., Bleidorn, W., Lamb, M. E., Gosling, S. D., \& Rentfrow, P. J. (2015). Geographically varying associations between personality and life satisfaction in the London metropolitan area. Proceedings of the National Academy of Sciences, 112(3), 725-730. https://doi.org/10.1073/pnas.1415800112

Karsai, M., Perra, N., \& Vespignani, A. (2014). Time varying networks and the weakness of strong ties. Scientific Reports, 4, 4001. https://doi.org/10.1038/srep04001

Kitayama, S., Ishii, K., Imada, T., Takemura, K., \& Ramaswamy, J. (2006). Voluntary settlement and the spirit of independence: Evidence from Japan's “northern frontier.” Journal of Personality and Social Psychology, 91(3), 369-384.

Kleinbaum, A. M., Jordan, A. H., \& Audia, P. G. (2015). An Altercentric Perspective on the Origins of Brokerage in Social Networks: How Perceived Empathy Moderates the SelfMonitoring Effect. Organization Science, 26(4), 1226-1242. https://doi.org/10.1287/orsc.2014.0961

Kuwabara, K., Hildebrand, C. A., \& Zou, X. (2018). Lay Theories of Networking: How Laypeople's Beliefs About Networks Affect Their Attitudes Toward and Engagement in Instrumental Networking. Academy of Management Review, 43(1), 50-64. https://doi.org/10.5465/amr.2015.0076 
Laurence, J. (2011). The Effect of Ethnic Diversity and Community Disadvantage on Social Cohesion: A Multi-Level Analysis of Social Capital and Interethnic Relations in UK Communities. European Sociological Review, 27(1), 70-89.

https://doi.org/10.1093/esr/jcp057

Laux, L., \& Renner, K.-H. (2002). Self-Monitoring und Authentizität: Die verkannten Selbstdarsteller. [Self-monitoring and authenticity: The misjudged self-presenters.]. Zeitschrift Für Differentielle Und Diagnostische Psychologie, 23(2), 129-148. https://doi.org/10.1024//0170-1789.23.2.129

Lennox, R. D., \& Wolfe, R. N. (1984). Revision of the Self-Monitoring Scale. Journal of Personality and Social Psychology, 46(6), 1349. https://doi.org/10.1037/00223514.46.6.1349

Lu, J. G. (2021). A social network perspective on the Bamboo Ceiling: Ethnic homophily explains why East Asians but not South Asians are underrepresented in leadership in multiethnic environments. Journal of Personality and Social Psychology. https://doi.org/10.1037/pspa0000292

Lu, J. G., Swaab, R. I., \& Galinsky, A. D. (2021). Global Leaders for Global Teams: Leaders with Multicultural Experiences Communicate and Lead More Effectively, Especially in Multinational Teams. Organization Science. https://doi.org/10.1287/orsc.2021.1480

Lukaszewski, A. W., Gurven, M., von Rueden, C. R., \& Schmitt, D. P. (2017). What Explains Personality Covariation? A Test of the Socioecological Complexity Hypothesis. Social Psychological and Personality Science, 8(8), 943-952. https://doi.org/10.1177/1948550617697175 
McPherson, M., Smith-Lovin, L., \& Brashears, M. E. (2006). Social Isolation in America: Changes in Core Discussion Networks over Two Decades. American Sociological Review, 71(3), 353-375. https://doi.org/10.1177/000312240607100301

Mitchell, C. U., \& LaGory, M. (2002). Social Capital and Mental Distress in an Impoverished Community. City \& Community, 1(2), 199-222. https://doi.org/10.1111/15406040.00017

Miville, M. L., Gelso, C. J., Pannu, R., Liu, W., Touradji, P., Holloway, P., \& Fuertes, J. (1999). Appreciating similarities and valuing differences: The Miville-Guzman UniversalityDiversity Scale. Journal of Counseling Psychology, 46(3), 291-307. https://doi.org/10.1037/0022-0167.46.3.291

Momennejad, I., Duker, A., \& Coman, A. (2019). Bridge ties bind collective memories. Nature Communications, 10(1), 1578. https://doi.org/10.1038/s41467-019-09452-y

Morrison, E. W. (2002). Newcomers' relationships: The role of social network ties during socialization. Academy of Management Journal, 45(6), 1149-1160. https://doi.org/10.2307/3069430

Muthukrishna, M., \& Henrich, J. (2016). Innovation in the collective brain. Philosophical Transactions of the Royal Society B: Biological Sciences, 371(1690), 20150192. https://doi.org/10.1098/rstb.2015.0192

Muthukrishna, M., Shulman, B. W., Vasilescu, V., \& Henrich, J. (2014). Sociality influences cultural complexity. Proceedings of the Royal Society B: Biological Sciences, 281(1774), 20132511. https://doi.org/10.1098/rspb.2013.2511 
Niedenthal, P. M., Rychlowska, M., \& Wood, A. (2017). Feelings and contexts: Socioecological influences on the nonverbal expression of emotion. Current Opinion in Psychology, 17, 170-175. https://doi.org/10.1016/j.copsyc.2017.07.025

Niedenthal, P. M., Rychlowska, M., Wood, A., \& Zhao, F. (2018). Heterogeneity of long-history migration predicts smiling, laughter and positive emotion across the globe and within the United States. PLOS ONE, 13(8), e0197651. https://doi.org/10.1371/journal.pone.0197651

Niedenthal, P. M., Rychlowska, M., Zhao, F., \& Wood, A. (2019). Historical Migration Patterns Shape Contemporary Cultures of Emotion. Perspectives on Psychological Science, 14(4), 560-573. https://doi.org/10.1177/1745691619849591

Oh, H., \& Kilduff, M. (2008). The ripple effect of personality on social structure: Selfmonitoring origins of network brokerage. Journal of Applied Psychology, 93(5), 11551164. https://doi.org/10.1037/0021-9010.93.5.1155

Oishi, S., \& Kesebir, S. (2012). Optimal Social-Networking Strategy Is a Function of Socioeconomic Conditions: Psychological Science. https://doi.org/10.1177/0956797612446708

Perry-Smith, J. E., \& Shalley, C. E. (2003). The Social Side of Creativity: A Static and Dynamic Social Network Perspective. The Academy of Management Review, 28(1), 89-106. https://doi.org/10.2307/30040691

Putnam, R. D. (2000). Bowling Alone: America’s Declining Social Capital. In L. Crothers \& C. Lockhart (Eds.), Culture and Politics: A Reader (pp. 223-234). Palgrave Macmillan US. https://doi.org/10.1007/978-1-349-62965-7_12 
Putterman, L., \& Weil, D. N. (2010). Post-1500 population flows and the long-run determinants of economic growth and inequality. The Quarterly Journal of Economics, 125(4), 16271682. https://doi.org/10.1162/qjec.2010.125.4.1627

R Development Core Team. (2008). R: A Language and Environment for Statistical Computing. R Foundation for Statistical Computing. http://www.R-project.org

Renner, K.-H., Enz, S., Friedel, H., Merzbacher, G., \& Laux, L. (2008). Doing as if: The histrionic self-presentation style. Journal of Research in Personality, 42(5), 1303-1322. https://doi.org/10.1016/j.jrp.2008.04.005

Riggio, R. E., \& Friedman, H. S. (1982). The interrelationships of self-monitoring factors, personality traits, and nonverbal social skills. Journal of Nonverbal Behavior, 7(1), 3345. https://doi.org/10.1007/BF01001776

Rivas, J., Hale, K., \& Burke, M. G. (2019). Seeking a sense of belonging: Social and cultural integration of international students with American college students. Journal of International Students, 9(2), 682-704.

Ruggles, S., Flood, S., Goeken, R., Grover, J., Meyer, E., Pacas, J., \& Sobek, M. (2020). IPUMS USA: Version 10.0 [dataset]. IPUMS.

Rychlowska, M., Miyamoto, Y., Matsumoto, D., Hess, U., Gilboa-Schechtman, E., Kamble, S., Muluk, H., Masuda, T., \& Niedenthal, P. M. (2015). Heterogeneity of long-history migration explains cultural differences in reports of emotional expressivity and the functions of smiles. Proceedings of the National Academy of Sciences, 112(19), E2429E2436. 
Sasovova, Z., Mehra, A., Borgatti, S. P., \& Schippers, M. C. (2010). Network Churn: The Effects of Self-Monitoring Personality on Brokerage Dynamics. Administrative Science Quarterly, 55(4), 639-670. https://doi.org/10.2189/asqu.2010.55.4.639

Shaffer, D. R., Smith, J. E., \& Tomarelli, M. (1982). Self-monitoring as a determinant of selfdisclosure reciprocity during the acquaintance process. Journal of Personality and Social Psychology, 43(1), 163-175. https://doi.org/10.1037/0022-3514.43.1.163

Shrira, I., Wisman, A., \& Noguchi, K. (2018). Diversity of historical ancestry and personality traits across 56 cultures. Personality and Individual Differences, 128, 44-48. https://doi.org/10.1016/j.paid.2018.02.013

Smaldino, P., Lukaszewski, A., Rueden, C. von, \& Gurven, M. (2018). Niche Diversity Can Explain Cross-Cultural Differences in Personality Structure. https://doi.org/10.31234/osf.io/53wxg

Smolla, M., \& Akçay, E. (2019). Cultural selection shapes network structure. Science Advances, 5(8), eaaw0609. https://doi.org/10.1126/sciadv.aaw0609

Snyder, M. (1974). Self-monitoring of expressive behavior. Journal of Personality and Social Psychology, 30(4), 526-537. https://doi.org/10.1037/h0037039

Snyder, M., \& Gangestad, S. (1986). On the nature of self-monitoring: Matters of assessment, matters of validity. Journal of Personality and Social Psychology, 51(1), 125.

Thomson, R., Yuki, M., Talhelm, T., Schug, J., Kito, M., Ayanian, A. H., Becker, J. C., Becker, M., Chiu, C., Choi, H.-S., Ferreira, C. M., Fülöp, M., Gul, P., Houghton-Illera, A. M., Joasoo, M., Jong, J., Kavanagh, C. M., Khutkyy, D., Manzi, C., ... Visserman, M. L. (2018). Relational mobility predicts social behaviors in 39 countries and is tied to 
historical farming and threat. Proceedings of the National Academy of Sciences, 115(29), 7521-7526. https://doi.org/10.1073/pnas.1713191115

Wilmot, M. P., DeYoung, C. G., Stillwell, D., \& Kosinski, M. (2016). Self-Monitoring and the Metatraits: Self-Monitoring and Metatraits. Journal of Personality, 84(3), 335-347. https://doi.org/10.1111/jopy.12162

Wilmot, M. P., Kostal, J. W., Stillwell, D., \& Kosinski, M. (2017). Using Item Response Theory to Develop Measures of Acquisitive and Protective Self-Monitoring From the Original Self-Monitoring Scale. Assessment, 24(5), 677-691. https://doi.org/10.1177/1073191115615213

Wood, A., Rychlowska, M., \& Niedenthal, P. M. (2016). Heterogeneity of long-history migration predicts emotion recognition accuracy. Emotion, 16(4), 413-420. https://doi.org/10.1037/emo0000137

Yang, Y. C., Boen, C., Gerken, K., Li, T., Schorpp, K., \& Harris, K. M. (2016). Social relationships and physiological determinants of longevity across the human life span. Proceedings of the National Academy of Sciences, 113(3), 578-583. https://doi.org/10.1073/pnas.1511085112

Zaidner, Y., Centi, L., Prévost, M., Mercier, N., Falguères, C., Guérin, G., Valladas, H., Richard, M., Galy, A., Pécheyran, C., Tombret, O., Pons-Branchu, E., Porat, N., Shahack-Gross, R., Friesem, D. E., Yeshurun, R., Turgeman-Yaffe, Z., Frumkin, A., Herzlinger, G., ... Hershkovitz, I. (2021). Middle Pleistocene Homo behavior and culture at 140,000 to 120,000 years ago and interactions with Homo sapiens. Science. https://doi.org/10.1126/science.abh3020 
Zimmermann, J., \& Neyer, F. J. (2013). Do we become a different person when hitting the road? Personality development of sojourners. Journal of Personality and Social Psychology, 105(3), 515-530. https://doi.org/10.1037/a0033019 